\title{
Adaptation of students to distance learning in COVID-19 conditions in terms of ultradian rhythms of the cardiovascular system
}

\author{
Tatyana Bashkireva ${ }^{1 *}$, Anastasia Bashkireva ${ }^{2}$, Alexander Morozov ${ }^{3}$, Alexander Severin $^{4}$, \\ Nadezhda Fateeva $^{5}$, Larisa Baykova ${ }^{1}$, and Evgeniya Severina ${ }^{4}$ \\ ${ }^{1}$ Institute of Psychology, Pedagogy and Social Work, Ryazan State University named after S.A. \\ Yesenin, associate professor, st. Svobody, 46, 390000, Ryazan, Russia \\ ${ }^{2}$ Faculty of Physical Education and Sports, Ryazan State University named after S.A. Yesenin, \\ professor, st. Svobody, 46, 390000, Ryazan, Russia \\ ${ }^{3}$ Research Center, The Federal State Institution "Research Institute of the Federal penitentiary service \\ of Russia," Narvskaya St., 15 A, b. 1, 125130, Moscow, Russia. \\ ${ }^{4}$ Faculty of Medicine, Peoples' Friendship University of Russia, st. Miklukho-Maklaya, 8, 117198, \\ Moscow, Russia \\ ${ }^{5}$ Institute of Psychology and Pedagogy, Tyumen State University, professor, st. Volodarsky, 6, \\ 625003 Tyumen, Tyumen region, Russia
}

\begin{abstract}
The emergence of the COVID-19 pandemic has led to the transition of educational organizations to self-isolation and distance learning. Once in the new conditions, it was necessary to adapt to the fact that the training format had changed. One of the important tasks for us was to study students' health and the peculiarities of their adaptation to the acquisition of knowledge and skills using information and communication technologies remotely. Based on Ryazan State University, studies carried out aimed at studying the adaptation and mental health of students to the conditions of distance learning during the period of COVID-19 in terms of ultradian rhythms of the cardiovascular system using the Belgian test "Reaction of the cardiovascular system to load in the form of inclinations torso. " The obtained biorhythmological data of health status according to the Belgian test showed that the health status of students in the conditions of distance learning in connection with the COVID-19 pandemic and selfisolation corresponded to the working state. Distance education satisfied introverts, not extroverts.
\end{abstract}

\section{Introduction}

Digital learning involves acquiring knowledge using Internet resources at a specified time or with its own choice. Distance learning considered to work in a digital space at a distance (remotely) on a leader's instructions. The definition of these concepts was given recently.

\footnotetext{
*Corresponding author: bashkirevat@bk.ru
} 
According to literature sources with access to distance learning and self-isolation in connection with the COVID-19 pandemic, some students experienced fear and anxiety when switching to online classes [1]. However, most did it quickly and in a short time. In general, according to F. Ainan et al., Everyone achieves appropriate adaptive models [ibid]. Researchers note the impact of the digital divide, which has shown that attitudes towards ICT depend on students' perception of the degree to which digital technologies affect learning and academic performance [6]. Teachers and educators' tendency to adhere to conservative views and not want to change their teaching practices $[4,7]$ often explain the slow adaptation of education to digital media.

Modern communication resources make it possible to create new learning systems through existing channels, allowing young people to develop their social, educational, and personal interactions [8]. In recent years, significant changes have occurred in Western society due to the widespread use of information and communication technologies and their increased use among adolescents.

Around the world, the outbreak of COVID-19 has destroyed many lives. The rapid increase in infections has created feelings of uncertainty and anxiety about what will happen. It also increases stress levels among teachers and students. This stress can lead to adverse effects on the learning and psychological health of students. Questions arise: Are universities taking proactive measures to support student mental health and well-being? Are there professionally trained counselors in universities who can understand these students?

High mortality rates because of the rapid spread of COVID-19 have begun to seriously threaten people's physical and mental health and cause some mental illnesses such as depression, post-traumatic stress disorder, anxiety, and panic disorder [6]. Depression is one of the most common infectious disease (EID) psychiatric disorders [2]. Recent studies have shown that during the COVID-19 outbreak, depression rates in the general population range from $3.7 \%$ to $48.3 \%$ and $6.46 \%-50.7 \%$ among healthcare workers in China [5].

Studies show that during the period of infectious diseases, students of the sports faculty are least susceptible to the psychological effects of the emerging quarantine conditions [9]. It may indicate that these students protected from boredom and sadness because they constantly contact physical activity and sports. It found in various studies that suggest that belonging to a sports environment helps people in their development [3].

The transition entirely to distance learning was new for teachers and students of higher education. Previously, some of this work carried out within the framework of traditional training using Moodle software. Once in the new conditions, it was necessary to adapt to the fact that the training format had changed. One of the important tasks for us was studying the health of students and the peculiarities of their adaptation to this format of education [7]. In science, the influence of digital education on the sensory and cardiovascular system, functional, mental, and psychological state, and students' cognitive activity not sufficiently studied.

\section{Method}

Based on Ryazan State University, studies carried out aimed to research students' adaptation and mental health to the conditions of distance learning during the period of COVID-19. In total, investigate 280 students of 1-4 courses. This paper presents the results of Bachelor graduates only. This is because April for graduates is associated with completing both the academic graduation year and the writing of the thesis.

This research was faced with the task of identifying how did distance learning in the context of the COVID-19 pandemic and self-isolation affects the health of students? How does the process of adaptation to such training take place? What feelings accompanied them 
in the process of educational activity? What is the opinion of students towards distance learning over a long period?

According to the assignment, during April, students took daily measurements of their condition according to the Belgian test, "The reaction of the cardiovascular system to a load in the form of torso bends." Despite the individual rhythm of work at the computer in the digital space, the time of measurements was regulated and was carried out during the daily maximum $(8.30 ; 16.30)$ and the daily minimum $(14.00,20.30)$ of mental performance.

Throughout the study, students were in self-isolation; during the study period, they recorded complaints of ailments in observation diaries. Students themselves regulated sleep time. In the group, it averaged about 8 hours. Working hours at the computer were determined individually.

The essence of the test is that: 1) it is necessary to count the pulse in 10 seconds at rest write down the result (P1);2) within one and a half minutes, perform 20 inclinations with lowering of the hands; 3 ) count the pulse for 10 seconds immediately after the exercise - write down the result $(\mathrm{P} 2)$; 4) rest for one minute and measure the pulse again for 10 seconds (P3); 5) calculate the indicator characterizing the state of the cardiovascular system according to the formula $(\mathrm{P} 1+\mathrm{P} 2+\mathrm{P} 3-33) / 10$. Following the methodology, the state of the cardiovascular system assessed: 0-0.3 - excellent; 0.31-0.6 - good; 0.61-0.9 - average; 0.911.2 - undesirable; more than 1.2 - unsatisfactory. The author has studied the indicators of ultradian (from minutes to 10-12 hours), rhythms of adaptive reactions of students' cardiovascular system to the load in conditions of distance learning.

\section{Results and discussion}

\subsection{Time spent in digital space and its impact on the cardiovascular system}

The study results showed that the maximum time of work in the digital space during the day was 12 hours, and the minimum time was 0 hours. More than 5 hours a day worked at a computer in the digital space $-53.6 \%$ of students. According to the Belgian test, the state of the cardiovascular system in this group corresponded to the average level $(0.79 \pm 0.16 ; \pm \sigma=$ 0.21). $28.6 \%$ of students worked in digital space remotely for 3 hours or less. Their health level also corresponded to the average level $(0.74 \pm 0.15 ; \pm \sigma=0.20)$.

When interviewing $21.4 \%$ of students, the author recorded the ultradian rhythm of the cardiovascular system according to the Belgian test coefficient from 0.95 to 1.3. This result is undesirable for the health of students and causes tension in the indicators of the functional state of the cardiorespiratory system. The author attributed this group to the risk group since, regardless of the number of hours spent in information and communication technologies, the cardiovascular system's indicators did not fall below the 0.95 coefficient.

\subsection{Study of ultradian rhythms according to the Belgian test}

The study of average monthly ultradian rhythms (from 8.30 to 20.30) showed that during the study period, the indicators at 8.30 were significantly higher than $14.00(\mathrm{r}=0.6 ; \mathrm{P}<0.37 \div$ $0.48 ; \mathrm{P}>0.05$ ) and corresponded to the average level, it shows in Figure 1. The minimum values corresponded to the daily minimum of mental activity, which indicates a favorable, efficient state of the body and a timely planned rest period. According to the Belgian test, we revealed fatigue and tension in the functional state in terms of the evening minimum. This indicates that students spend most of their time in ICT conditions after the second daily maximum (i.e., after 16.30). 


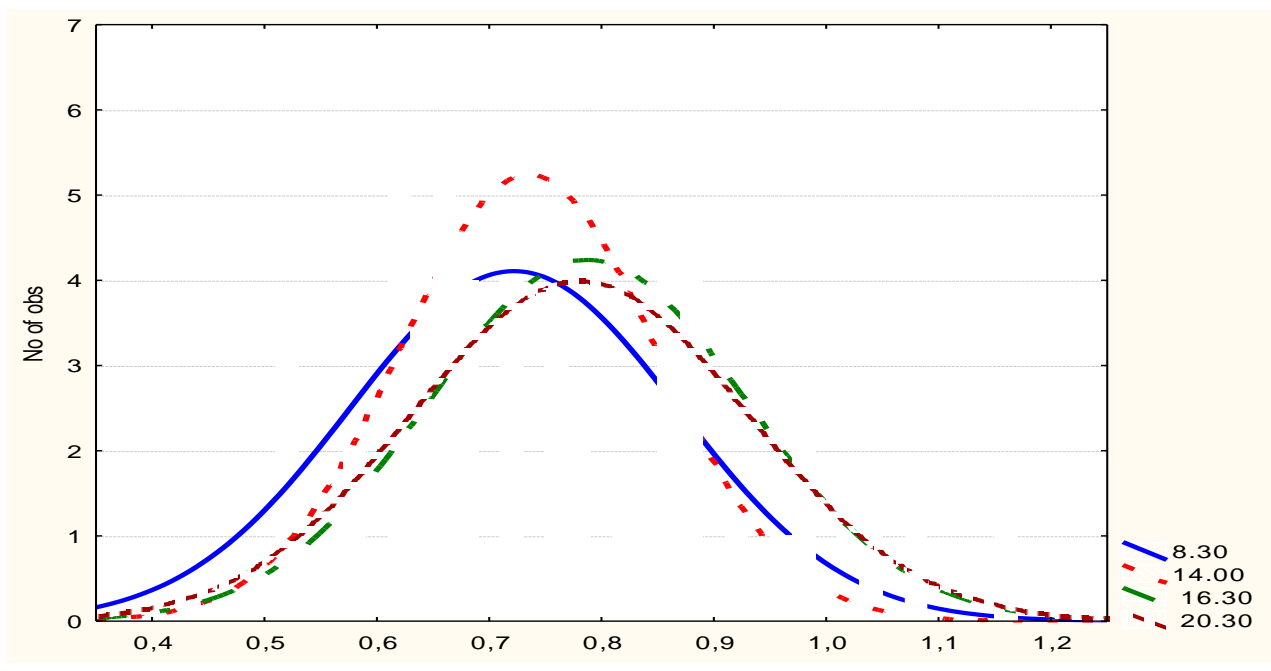

Fig. 1. Average monthly ultradian rhythms of the cardiovascular system according to the Belgian test in students in the context of the COVID-19 pandemic and distance digital learning.

\subsection{Health status of students in distance learning during the Covid-19 period}

In general, the results of ultradian rhythms in terms of the cardiovascular system showed that students' body during the study period was in the range of the average level. The data obtained can conclude that students' state of health in the conditions of distance learning in connection with the COVID-19 pandemic and self-isolation corresponded to the working state. Of interest is the opinion of the students who took part in the study. Here are some conclusions that students came to because of the analysis of the information they received:

1. Based on the results of the study "Student health in digital distance education", the author can conclude that the work of my cardiovascular system is at a high level. However, compared to traditional education methods, distance learning can lead to visual impairment. It is necessary to carry out scientific research to regulate the time of work in the digital space, depending on the age of the person.

2. I liked the work in remote mode. Habitual communication in university classrooms was still lacking.

3. The state of health in terms of the cardiovascular system, using the Belgian test, was in the range of 0.6-1.2 - from medium to undesirable. The average daily rate for the entire observation period was 0.91 (this is the upper limit of the average state of the cardiovascular system on the border with undesirable). The results of my observation showed that changes in the indicators of the cardiovascular system are cyclical. A relative improvement followed by a deterioration in the indicator. The general state of the clock over the entire observation period fluctuated in an insignificant range. However, it worsened daily towards evening (20:30). The time spent at the computer during the month ranged from two to 9 hours. I liked distance learning because it was possible to get enough sleep and independently determine the time of intensive work. There was time for selfdevelopment.

4. Analyzing the received data showed that in the evening hours (20:30) the pulse is very high. I think this is because in the evening I am most physically active. I do not think the amount of computer works greatly affected my health during distance learning. Approximately the same amount of time devoted to distance learning. I believe that 
distance learning is advisable to use in education. However, the technical improvement of educational platforms needed.

5. The totality of all the data obtained indicates 1) there was a deterioration in the state of the cardiovascular system (peak at 14:00) and the best - in the evening $(20: 30) ; 2$ ) in general, distance learning and the prevailing sedentary lifestyle at the computer, which is typical for it, did not have a big impact on the state of my cardiovascular system. I believe that distance learning used in combination with traditional education. There is more time for self-development.

Throughout the study, there are differences between morning and evening heart rate measurements. These differences may influence by working at the computer during distance learning. The main time spent at the computer fell on lunch and evening hours. Distance learning showed that I do not know how to organize my way of independent work. I need to study. According to the Belgian test, the obtained biorhythmological data of the state of health showed that $92.1 \%$ of students had an organism in the range of $0.61-0.9$, which corresponds to the average level.

\section{Conclusion}

In general, the students assessed their health status positively during the period of distance learning in self-isolation. $83.7 \%$ of the surveyed acquired a positive experience of distance learning. There were no complaints of negative mental state or depression. All students noted the difficulties of adapting to this teaching format in the early days. However, already from the second week, they did not have trouble. The time of awakening was especially satisfying since there was an opportunity to get enough sleep. In the traditional learning model, everyone stressed by getting up early and transport time. Only 54.8\% spend more than 2 hours traveling to and from the university.

During the period of distance learning in the context of the COVID-19 pandemic, our questioning showed that $50 \%$ of students expressed satisfaction with distance learning, explaining their opinion with a high level of opportunities for the independent search for the necessary information, great opportunities for self-development, self-realization, and selfimprovement. Fifty percent of the students also expressed the "eye to eye" learning deficit. Among the difficulties of distance learning, they indicated difficulties in finding and analyzing information, communicative dissatisfaction, and the need for approval. According to our observations, distance learning has satisfied introverts and is not satisfied - extroverts.

Thus, according to the Belgian test, the analysis of ultradian rhythms among students showed that not all students had formed an effective individual style of learning activity. Besides, the time of independent work in ICT determined by them was noneffective and the most favorable for their body. During the period of study of students at the university, it is necessary to pay attention to the support of the individual style of activity and its formation.

\section{References}

1. A. Al-Rabiaahab, MH Temsahabc, AA Al-Eyadhy et al, Middle East Respiratory Syndrome Corona Virus (MERS-CoV) associated stress among medical students at a university teaching hospital in Saudi Arabia, in J Infect Public Health (2020) [Epub ahead of print] https://doi.org/10.1016/j.jiph.2020.01.005

2. A. Caspi, N. Bolger, J. Eckenrode, Linking person and context in the daily stress process, in J. Abnorm. Psychol. Soc. Psychol 52 (1987)

3. Li Chen, Timeout decisions of basketball coaches of men's and women's collegiate teams (1996) 
4. I.W. Mak, C.M. Chu, P.C. Pan, M.G. Yiu, V.L. Chan, Long-term psychiatric morbidities among SARS survivors, in Gen. Hosp. Psychiatry. 31, 4 (2009) https:// doi.org/10.1016/j.genhosppsych.2009.03.001.

5. Sahu Pradeep, Closure of Universities Due to Coronavirus Disease 2019 (COVID-19): Impact on Education and Mental Health of Students and Academic Staff in Cureus. 12, 4 https://doi.org/10.7759/cureus.7541

6. Shock, fear, and fatalism: as coronavirus prompts colleges to close, students grapple with uncertainty. (2020). Accessed: March 12, 2020:

https://www.chronicle.com/article/ShockFearFatalism-As/248240

7. J. Sweller, Implications of cognitive load theory for multimedia learning, in R.E. Mayer (Ed.), The Cambridge handbook of multimedia learning. New York: Cambridge University Press (2005)

8. H.C. Triandis, M.J. Gelfand, Converging measurement of horizontal and vertical individualism and collectivism, in J. Abnorm. Soc. Psychol. 74, 1 (1998)

9. V. Vesterinen, K. Häkkinen, T. Laine, E. Hynynen, J. Mikkola, A. Nummela, Predictors of individual adaptation to high-volume or high-intensity endurance training in recreational endurance runners, in Scand J Med Sci Sports. 6, 8 (2016) 